\title{
Comparative Study of DSP Techniques for the Effective Modeling and Design of Highly Complex RF-MEMS Structures
}

\author{
Jong-Hoon Lee, Nathan Bushyager, and Manos M. Tentzeris \\ Georgia Electronic Design Center, School of Electrical and Computer Engineering \\ Georgia Institute of Technology, Atlanta GA 30332-0269 USA \\ Fax:(404) 894-0222, Email:jonglee@ece.gatech.edu
}

\begin{abstract}
Four DSP-based digital predictors (Prony's, covariance, forward-backward, matrix pencil), that are commonly used to enhance the time-domain modeling and design of highly complex RF MEMS structures, are evaluated in terms of computational efficiency and accuracy as a function of the model order, the decimating factor, and the size of sample train. For a benchmarking case of an RF MEMS tuner, it is found that while covariance method has the best performance in terms of accuracy matrix pencil method confers robustness to computational economies (less numerical effort) and saves more CPU time with a smaller model order that can be selected by easy and efficient criteria.
\end{abstract}

Index Terms - Digital signal processing (DSP), predictors, Prony, covariance, forward-backward, matrix pencil, FDTD, Time-Domain Modeling, RF-MEMS.

\section{INTRODUCTION}

The finite-difference time-domain (FDTD) scheme is one of the most powerful and versatile techniques used for numerical simulations [1], since it provides accurate solutions of Maxwell's equations for a wide frequency range with a single run while avoiding oversimplifying approximations. Nevertheless, the addition of complex metal and dielectric shapes (antennas, multilayer passives, MEMS membranes) and realistic material characteristics (metal finite conductivity and thickness) into simulation lead to very computationally intensive FDTD simulations [2]. Also, the time-step of the simulations is limited by the smallest feature of the device being modeled, something very important especially for resonant structures that require hundreds of thousands or even millions of time-steps. Most finely detailed RF-MEMS (membrane) structures are combined into circuits containing large connecting structures, thus requiring a very large number of time-steps. To alleviate this problem, there is a need for the hybridization of FDTD with digital signal processing (DSP) techniques that are robust against system dynamics and accurately predict the late time response and frequency behaviors of the system from a relatively short sample range, small decimating factor, and small model order. A number of alternative DSP predictors has been investigated by several authors to overcome the computational overhead for microwave circuits [3]-[7] with Prony's [3]-[4], autoregressive (AR) models (covariance, forwardbackward) [5]-[6], and matrix pencil method [7] being the most popular ones. However, each technique has different operational characteristics and tradeoffs, thus making it difficult to choose the best predictor for each specific application. In this letter, these four digital predictors are benchmarked for a representative geometry of an RF MEMS $2 \times 2$ bit tuner and their computational economies and accuracy are discussed in terms of simplicity, model order, decimating factor, and the size of sample train.

\section{Digital Signal Processing Predictors}

Prony's method models sampled data as a linear combination of complex exponentials [1] and consists of a multi-step procedure. The major problem of Prony's method is the model order selection that determines the quality of the spectral resolution and the appearance of spurious modes [1]. The model order can be decided by the Minimum Description Length (MDL) choosing minimum error between the model and the data samples. On the other hand, the AR models (covariance, forwardbackward) are based on the source-filter model that is constrained to be all-pole linear filter. This amounts to performing a linear prediction of the next sample as a weighted sum of past samples. Forward-backward method is less sensitive to model order than covariance since it uses only time-data, not covariance values that are approximated with inaccurate functions of the known time signal [1]. Since AR models are purely pole linear systems, they require a large number of poles to synthesize the dominant zero of systems, thus possibly leading to large-order AR models. MDL that is regarded as a consistent estimator of order compared to AIC that often overestimates the model order [3] is used to choose the model order. The Matrix pencil method also approximates the early FDTD response as a sum of damped complex exponentials and extrapolates the late 
transient response by summing complex exponentials with complex coefficients obtained by singular-value decomposition (SVD) and least-squares algorithm [7]. The major difference with Prony's method and AR models is the formulation of eigenvalue problems to determine the poles. In this way, the model order can be simply estimated to be the number of largest singular values from SVD of matrices composed of data vectors of noiseless signal.

\section{COMPARATIVE EVALUATION}

The performance of the above four predictors was evaluated for the benchmarking 2x2 RF-MEMS tuner geometry shown in figure 1(a). This device is used to match numerous impedances over a wide frequency band by using MEMS capacitive switches built on membranes (Fig. 1 (b)) to connect or disconnect 4 shunt capacitive stubs providing $2^{4}=16$ impedance matching combinations. The structure was excited with a Gaussian derivative pulse in time, with a maximum frequency of $25 \mathrm{GHz}$ in FDTD (size of variable FDTD grid \{nx: 372, ny: 60, nz: $318\}$; range of cell sizes $\{\mathrm{dx}: 24.35 \mu \mathrm{m} \sim 50 \mu \mathrm{m}, \mathrm{dy}: 4 \mu \mathrm{m}$ $\sim 30 \mu \mathrm{m}, \mathrm{dz}: 22.2 \mu \mathrm{m} \sim 52 \mu \mathrm{m}\})$. Without loss of generality, the results for 0001 ("1-OFF 2-OFF 3-OFF 4-ON") were chosen as the example for the timedomain performance of the DSP predictors.

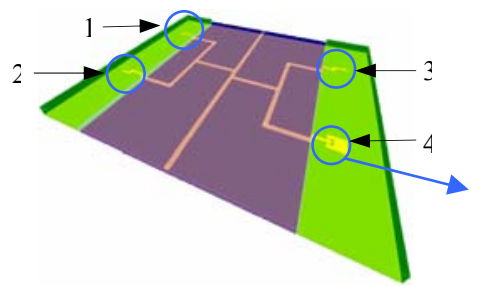

(a)

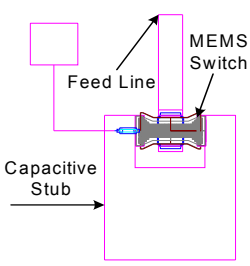

(b)
Figure 1. (a)Diagram of simulated '2-bit $x$ 2bit' RF-MEMS tuner and (b) RF-MEMS capacitive stub

Figure 2 shows the procedure of the separation of the predictor-"training" time-sequence and of the predicted late transient time-steps. The first 100,000 time steps dominated by excitation and early high-oscillating transient data should be discarded. The period between 100,000 to 130,000 was used for building the model that was tested in terms of approximation error in the timeinterval between 130,000 to 160,000 steps, which was decided by finding out the minimum range of the period that behaves repeatedly over late transient response. Since the decay of late transient response is slow, good results could be produced from the prediction of a relatively large number of samples. In the simulated case, the predictors were used to extrapolate the time sequence from 130,000 up to 200,000 and the numerical error is evaluated with respect to results evaluated with direct application of FDTD.

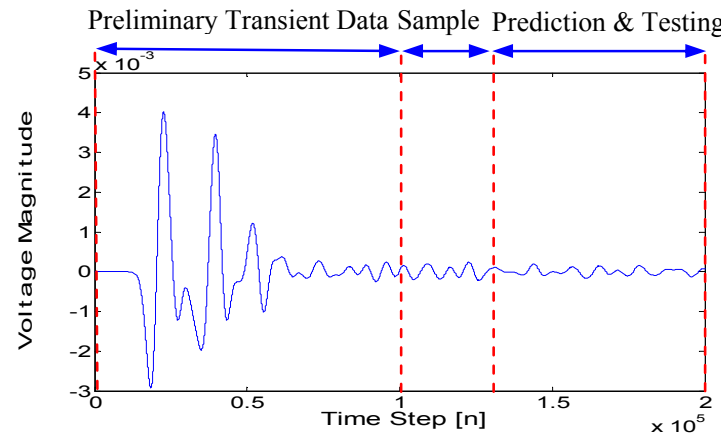

Figure 2. The FDTD time response of 0001 impedance matching of RF MEMS tuner.

Figure 3 (a) displays the waveform of the direct FDTD computation and the extrapolated waveform by FDTD plus Prony's method at the output port of RF-MEMS tuner. The signal was decimated by 100 and the $66^{\text {th }}$ model order was used for prediction. The decimating factor was selected by the ratio of Nyquist frequency $\left(\mathrm{f}_{\mathrm{n}}=(2 \Delta \mathrm{t})^{-1}=220,781 \mathrm{GHz}\right)$ and maximum frequency $\left(\mathrm{f}_{\max }=25 \mathrm{GHz}\right)$ [4]. To avoid undesirable aliasing, decimating factor was set to $0.9\left(\mathrm{f}_{\mathrm{n}} / \mathrm{f}_{\max }\right)$ first then optimized to $0.12\left(\mathrm{f}_{\mathrm{n}} / \mathrm{f}_{\max }\right)$. It is observed that Prony's method produces very poor results for predicting the large amount of time steps of transient waveforms for highly resonant structures.

Both AR linear predictors were tested on the same data and displayed in figure 3 (b). Covariance method with the optimal decimating factor of 100 and the model order of 65 predicted the results of Fig. 3 (b). Very good corroboration between direct FDTD and FDTD plus Covariance is observed for voltage signatures. Also, covariance outperforms Prony's with same decimating factor and almost same model order (65 vs. 63) in voltage signature. Then forward-backward (FB) method was applied with the optimized $80^{\text {th }}$ model order and 50 decimating factor as presented in Fig. 3 (b). Although the performance of the forward-backward method is not better than the covariance method due to the higher order (80 vs. 65 ), less decimating factor (50 vs. 100) and more deviated matches, forward backward is much more stable than covariance with the change of parameters (decimating factor, model order). Finally, the matrix pencil (MP) technique was demonstrated (Fig. 3(d)) with 600 data obtained by 50 decimating factor and $46^{\text {th }}$ model order. Although MP method takes less numerical effort than Prony's and AR models, it provides a robust and accurate match in time domain with a lower model order than any other predictors. S21 spectrums of FDTD alone and FDTD plus covariance's method, carried out by fast Fourier transform (FFT) algorithm, are shown in figure 4 that represents the prediction performance of all extrapolated models in frequency domain. Good agreement could be occurred because most important spectral content is included in first 10,000 time steps so that discrepancies of four 
techniques are extremely small in the frequency domain. Nevertheless, it is observed a little difference around dips of response. FFT is mutual to the product of total number of iterations and the time step size therefore predicted value having less number of iteration could miss some of zero series that could affect this difference. A computational acceleration by $50 \%$ is achieved to obtain S21 spectrums with very good correlation to the direct FDTD application. Table I summarizes the numerical results of four DSP predictors. The Mean-square error (MSE) at the last row of the table can be used to evaluate the performance of the four techniques.
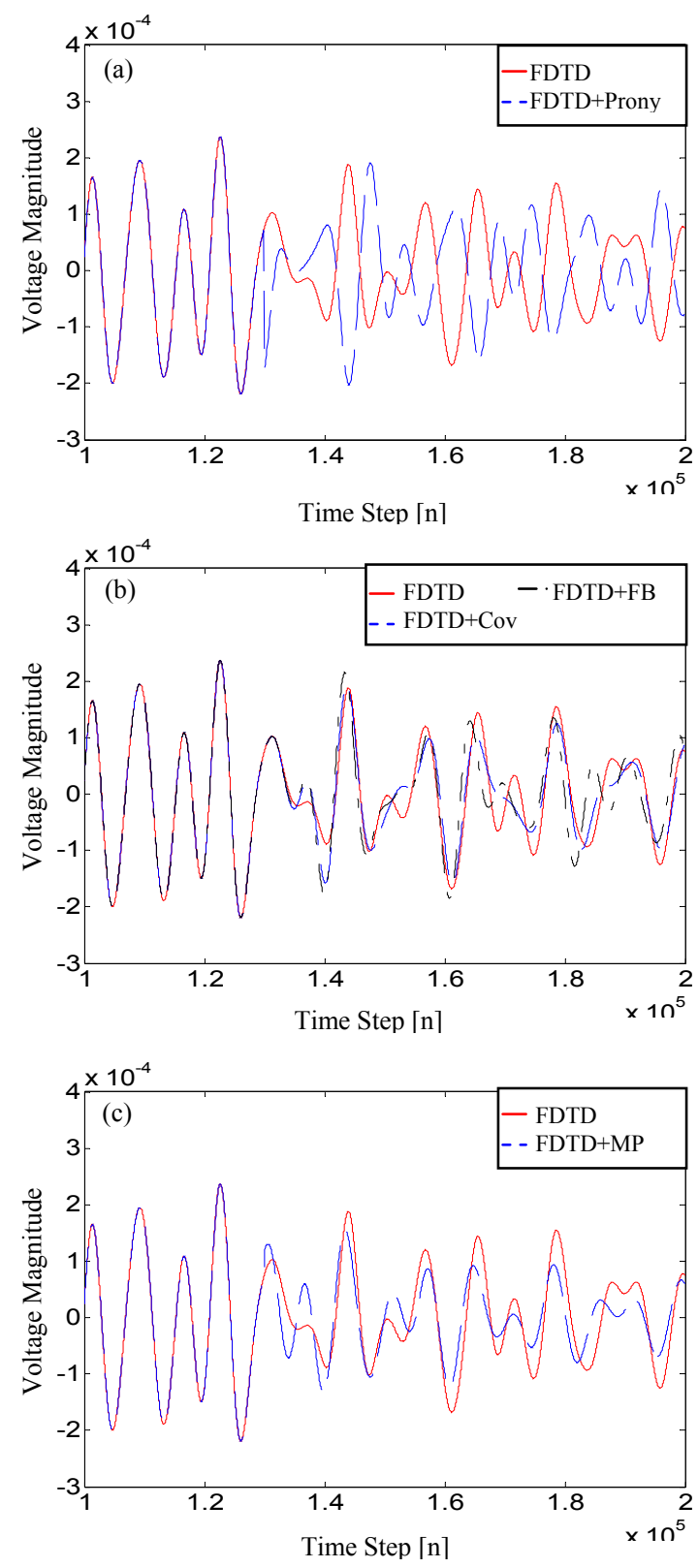

Figure 3. FDTD time sequences of RF-MEMS tuner FDTD alone (solid) (a) vs. FDTD + Prony's (dash), (b) vs. FDTD + covariance (dash) and vs. FDTD + FB (dashdot), (c) vs. FDTD + MP (dash)

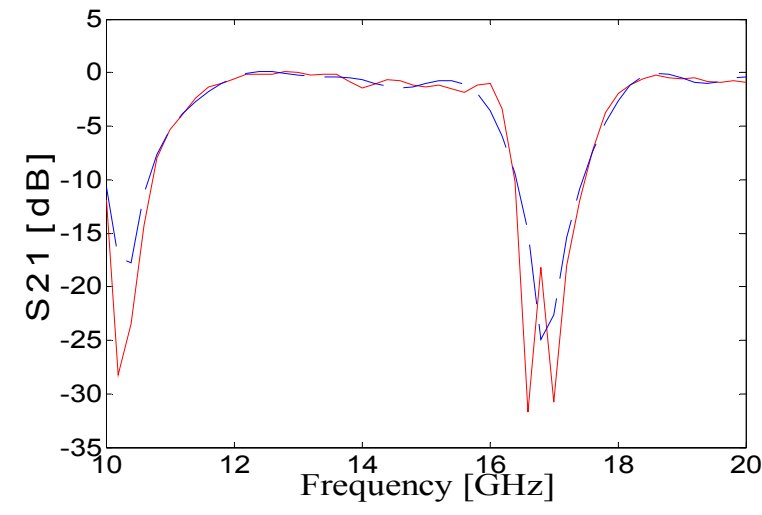

Figure 4. Compared S21: FDTD (solid), FDTD + Covariance (dashed)

TABLE I

SUMMARY OF NUMERICAL RESULTS

\begin{tabular}{|c|c|c|c|c|}
\hline $\begin{array}{c}\text { Predictor } \\
\text { Techniques }\end{array}$ & $\begin{array}{c}\text { Prony's } \\
\text { Method }\end{array}$ & $\begin{array}{c}\text { Covariance } \\
\text { Method }\end{array}$ & FB Method & $\begin{array}{c}\text { MP } \\
\text { Method }\end{array}$ \\
\hline $\begin{array}{c}\text { Sampling } \\
\text { Range }\end{array}$ & $\begin{array}{c}100,000- \\
130,000\end{array}$ & $\begin{array}{c}100,000- \\
130,000\end{array}$ & $\begin{array}{c}100,000- \\
130,000\end{array}$ & $\begin{array}{c}100,000- \\
130,000\end{array}$ \\
\hline $\begin{array}{c}\text { Sampling } \\
\text { Rate }\end{array}$ & 100 & 100 & 50 & 50 \\
\hline Model Order & 63 & 65 & 80 & 46 \\
\hline $\begin{array}{c}\text { MSE } \\
\text { (Time Probe/ } \\
\text { S-parameter) }\end{array}$ & $\begin{array}{c}1.6751 \mathrm{e}-8 \\
2.6776 \mathrm{e}- \\
4\end{array}$ & $\begin{array}{c}5.4863 \mathrm{e}-10 / \\
2.6721 \mathrm{e}-4\end{array}$ & $\begin{array}{c}2.0178 \mathrm{e}-9 / \\
2.6725 \mathrm{e}-4\end{array}$ & $\begin{array}{c}1.3909 \mathrm{e}-9 / \\
2.6724 \mathrm{e}-4\end{array}$ \\
\hline
\end{tabular}

Based on the simulation results from the benchmarking geometry, the covariance method that has the lowest MSE out of the four evaluated techniques, as well as the MP technique which has the simplest and more computationally effective implementation, were used to determine the other fifteen impedances that the tuner can match and the bandwidth of these matches, showing similar results. A plot of the matched impedances, both simulation and measurement is presented in figure 5. The simulation results are within $10 \%$ of the measurement results.

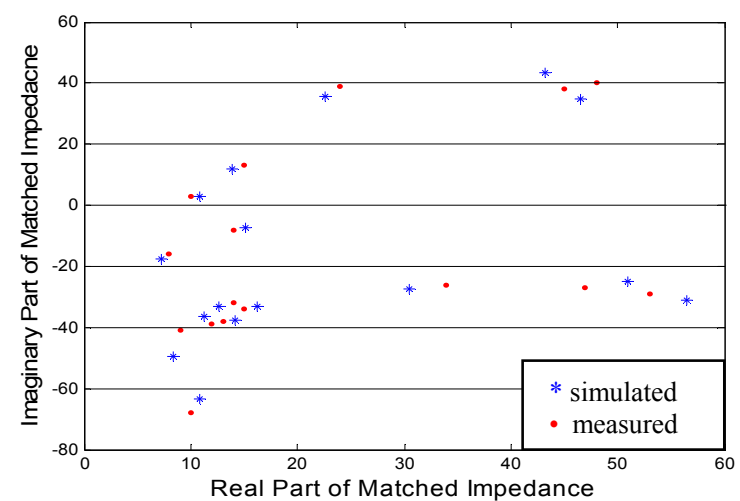

Figure 5. Plot of measured and simulated matched impedances at $20 \mathrm{GHz}$

\section{CONCLUSION}

Four DSP-based digital predictors (Prony's, covariance, forward-backward, matrix pencil) have been evaluated in terms of computational efficiency, 
implementation simplicity and accuracy for the timedomain modeling of a complex RF MEMS benchmarking geometry. It has been found that while covariance method performs as the best in terms of accuracy, the matrix pencil method confers robustness to computational economies (less numerical effort) and saves more CPU time with a smaller model order that can be selected by simple and efficient criteria. This technique could be effectively used for the accelerated design and optimization of RF MEMS structures with full-wave time-domain simulators such as FDTD and TLM.

\section{ACKNOWLEDGEMENT}

The authors wish to acknowledge the support of the Georgia Tech., Packaging Research Center, the Georgia Electronic Design Center, the NSF CAREER Award \#ECS-9984761 and the NSF Grant \#ECS-0313951. They would also like to thank Prof. J.Papapolymerou for providing the measurements for the RF tuner and F.Coccetti and Prof. P.Russer for their useful discussions on the matrix pencil technique.

\section{REFERENCES}

[1] A.Taflove and S.Hagness, Computational Electrodynamics: The Finite Difference Time Domain Method, $2^{\text {nd }}$ ed., Norwood, MA/U.S.A: Artech House, 2000.

[2] N.Bushyager, K.Lange, M. Tentzeris and J.Papapolymerou, "Modeling and Optimization of RF-MEMS Reconfigurable Tuners with Computational Efficient Time-Domain Techniques," in 2002 IEEE MTT-S Int. Microwave Sym. Dig, Seattle. WA, June 2002, pp. 883-886.

[3] W.L.Ko and R.Mittra, "A Combination of FDTD and Prony's Methods Analyzing Microwave Integrated Circuits," IEEE trans. on Microwave Theory and Techniques, vol. 39, pp. 2176-2181, Dec. 1991.

[4] K.Naishadham and X.Lin, "Application of Spectral Domain Prony's Method to the FDTD Analysis of Planar Microstrip Circuits," IEEE trans. on Microwave Theory and Techniques, vol 42, pp 2391-2398, Dec. 1994.

[5] J.Chen, et al., "Using Linear and Nonlinear Predictors to Improve the Computational Efficiency of the FDTD Algorithm," IEEE trans. on Microwave Theory and Techniques, vol. 42, pp. 19921997, Oct. 1994.

[6] V.Jandhyala, E.Michielssen and R.Mittra, "FDTD Signal Extrapolation Using the Forward-Backward Autoregressive(AR) Model," IEEE Microwave and Guided Wave letter, vol. 4, pp. 163-165, June 1994.

[7] Y.Hua and T.K.Sarkar, "Matrix Pencil Method for Estimating Parameters of Exponentially Dampled/Undamped Sinusoids in Noise," IEEE trans. on Acoust., Speech, and Signal Processing, vol. 38, pp. 814-824, May 1990. 